\title{
Effect of hyperviscosity on the geometry of the vorticity
}

\author{
Abdelhafid Younsi
}

\begin{abstract}
We consider the two-dimensional modified Navier-Stokes equations by adding a $p$-Laplacian. We provide upper bounds on the Hausdorff length of the level sets of the vorticity. We express them in terms of the Landau-Lifschitz estimates of the number of degrees of freedom in turbulent flow. The proof, based on very standard methods, relies on a straightforward relation between the size of the nodal set of the vorticity and the growth of hyperdissipation.
\end{abstract}

Mathematics Subject Classification. 35K55, 35Q30, 76D05, 76F20, 76F70.

Keywords. Navier-Stokes equations, Hyperviscosity, Vorticity, Turbulence, Hausdorff measure.

\section{Introduction}

In this paper, we provide upper bounds on the size of the nodal set $N_{\omega}^{0}=$ $\left\{x \in \Omega \subset \mathbb{R}^{2} / \omega(x, t)=0\right\}$ of the vorticity $\omega$ for solutions of modified 2D periodic Navier-Stokes equation. We consider a "regularized" Navier-Stokes system was proposed by Lions [18], who added an artificial hyper-viscosity term to the Navier-Stokes equations

$$
\begin{aligned}
\frac{d u}{d t}+\varepsilon(-\triangle)^{p} u-\nu \triangle u+(u . \nabla) u & =f(x), \quad \text { in } \Omega \times(0, \infty) \\
\operatorname{div} u & =0, \quad \text { in } \Omega \times(0, \infty), \\
u\left(x+L e_{i}, t\right) & =u(x, t) \quad i=1, \ldots, d \quad t \in(0, \infty) \\
u(x, 0) & =u_{0}(x), \quad \text { in } \Omega,
\end{aligned}
$$

on $\Omega=(0, L)^{d}$ with periodic boundary conditions and $\left(e_{1}, \ldots, e_{d}\right)$ is the natural basis of $\mathbb{R}^{d}$. Here $\varepsilon>0$ is the artificial dissipation parameter and $\nu>0$ is the kinematic viscosity of the fluid, $p>1$. The function $u$ is the velocity vector field, and $f$ is a given force field. For $\varepsilon=0$, the model is reduced to the Navier-Stokes system. 
Mathematical model for such fluid motion has been used extensively in turbulence simulations (see e.g. [7]) also see Borue and Orsag [2,3]. For further discussion of theoretical results concerning (1.1), see $[1,18,24]$.

For such a modified problem considered in a bounded domain, Lions was able to prove (cf. [18, Chap.1, Remarque 6.11]) the existence of a unique regular solution provided $(p \geq(d+2) / 4$ for the $d$-dimensional problem).

In the work [24], the strong convergence of the solution of this problem to the solution of the conventional system as the regularization parameter goes to zero, was established in each dimension $d \leq 4$.

For the Navier-Stokes system weak solutions of problem are known to exist by a basic result by Leray from 1934 [16], only the uniqueness of weak solutions remains as an open problem in 3D. Then the known theory of global attractors of infinite dimensional dynamical systems is not applicable to the 3D Navier-Stokes system.

In particular, in case one accepts the point of view that the dimension of a global attractor for the Navier-Stokes equations is associated with the number of degrees of freedom in turbulent flows Temam [23], then the Hausdorff measure $\mathcal{H}_{p}^{1}\left(N_{\omega}^{0}\right)$ is an important way to the understanding of turbulence theory [24].

Turbulence is a really hard problem. In this paper, we use analytic methods to describe and analysis the effect of hyper viscosity on turbulent flow. We are interested, in particular, in the treatment of the force term and the connections with the turbulence. Specifically, we prove this result, without using the Dirichlet or Rayleigh quotient [13].

Let $P_{m}$ be the projection onto the first $m$ eigenspaces of the Stokes operator $A=-\triangle$ and let $N_{\omega}^{0}=\left\{x \in \Omega \subset \mathbb{R}^{2} / \omega(x, t)=0\right\}$ the nodal sets of the vorticity $\omega$ for solutions of the Eq. (1.1). We provide an upper bound on the size of the nodal sets $\mathcal{H}_{p}^{1}\left(N_{\omega}^{0}\right)$ and we show that, the bounds necessarily depend on $m$ and $p$ this dependence is a fractional power of $p$. We also obtain here scale-invariant estimates on the one-dimensional Hausdorff measure $\mathcal{H}_{p}^{1}\left(N_{\omega}^{0}\right)$ in terms of the Landau-Lifschitz theory of the number of degrees of freedom in turbulent flow. Since expressing the above estimates in terms of the (dimensionless) Grashoff number $G_{r}$.

We find here that the upper bounds on the Hausdorff length of $N_{\omega}^{0}$ converge to the corresponding upper bounds of the usual 2D Navier-Stokes as $p=1$ and under certain hypothesis we recover the estimates for the usual 2D Navier-Stokes equations with potential force [13].

This result extends the work in [13] to the Navier-Stokes equations with general forcing modified by $p$-Laplacian. Related results for the 3D NavierStokes equations (with general forcing) can be found in [4]. The upper bounds on the Hausdorff measures of the level sets associated with solutions of some other partial differential equations were obtained in $[5,9,10,15,17]$.

The paper is organized as follows. In Sect.2, we present the relevant mathematical framework for the paper. In Sect. 3 , we provide upper bounds for the one-dimensional Hausdorff measure $\mathcal{H}_{p}^{1}$ of the level sets associated with the vorticity of the Navier-Stokes system with hyperdissipation. 
The question of the Hausdorff length of the level sets of the vorticity of solutions is thus completely answered for the two dimensional case. Our future investigations will be concerned with the corresponding problem for the three dimensional case. We have already reported partial results in this direction in Sect. 2 and in [24], where it was shown that the weak solution of the 3D regularized system converges strongly to a solution of the conventional system under certain conditions.

The methods validated in this paper certainly give us guidelines to elaborate the general three dimensional case.

\section{Notations and preliminaries}

In this section we introduce notations and the definitions of standard functional spaces that will be used throughout the paper. We denote by $H_{p e r}^{m}(\Omega)$, the Sobolev space of periodic functions. These spaces are endowed with the inner product

$$
(u, v)=\sum_{|\beta| \leq m}\left(D^{\beta} u, D^{\beta} v\right)_{L^{2}(\Omega)} \text { and the norm }\|u\|_{m}=\sum_{|\beta| \leq m}\left(\left\|D^{\beta} u\right\|_{L^{2}(\Omega)}^{2}\right)^{\frac{1}{2}} .
$$

We define the spaces $V_{m}$ as completions of smooth, divergence-free, periodic, zero-average functions with respect to the $H_{p e r}^{m}$ norms. $V_{m}^{\prime}$ denote the dual space of $V_{m}$ and $V$ denote the space $V_{0}$.

Let $P$ be the orthogonal projection in $L_{\text {per }}^{2}\left(\mathbb{R}^{2}\right)^{2}$ with the range $V$.

Let $A=-P \triangle$ the Stokes operator. It is easy to check that $A u=-\triangle u$ for every $u \in D(A)$. We recall that the operator $A$ is a closed positive self-adjoint unbounded operator.

The eigenvalues of $A$ are $\left\{\lambda_{j}\right\}_{j=1}^{j=\infty}, 0<\lambda_{1} \leq \lambda_{2} \leq \ldots$ and the corresponding orthonormal set of eigenfunctions $\left\{w_{j}\right\}_{j=1}^{j=\infty}$ is complete in $V_{0}$

$$
A w_{j}=\lambda_{j} w_{j}, \quad w_{j} \in D(A), \forall j .
$$

The spectral theory of $A$ allows us to define the powers $A^{p}$ of $A$ for $p \geq 1, A^{p}$ is an unbounded self-adjoint operator in $V_{0}$ with a domain $D\left(A^{p}\right)$ dense in $V_{2} \subset V_{0}$. We set here

$$
A^{p} u=(-\triangle)^{p} u \quad \text { for } \quad u \in D\left(A^{p}\right)=V_{2 p} .
$$

The space $D\left(A^{p}\right)$ is endowed with the scalar product and the norm

$$
(u, v)_{D\left(A^{p}\right)}=\left(A^{p} u, A^{p} v\right), \quad\|u\|_{D\left(A^{p}\right)}=\left\{(u, v)_{D\left(A^{p}\right)}\right\}^{\frac{1}{2}} .
$$

In the case for $p>0$, we have $D\left(A^{p}\right)=\left\{u \in V, \sum_{j=1}^{\infty} \lambda_{j}^{2 p}\left(u, w_{j}\right)^{2}<\infty\right\}$. For $p \in \mathbb{R}$ the scalar product and the norm in (2.1) can be written alternatively as

$$
(u, v)_{D\left(A^{p}\right)}=\sum_{j=1}^{\infty} \lambda_{j}^{2 p}\left(u, w_{j}\right)\left(v, w_{j}\right), \quad\|u\|_{D\left(A^{p}\right)}=\left\{\sum_{j=1}^{\infty} \lambda_{j}^{2 p}\left(u, w_{j}\right)\right\}^{\frac{1}{2}}
$$


and for $u \in D\left(A^{p}\right)$ we can write

$$
A^{p} u=\sum_{j=1}^{\infty} \lambda_{j}^{p}\left(u, w_{j}\right) w_{j} .
$$

Let us now define the trilinear form $b(\cdot, \cdot, \cdot)$ associated with the inertia terms

$$
b(u, v, w)=\sum_{i, j=1}^{3} \int_{\Omega} u_{i} \frac{\partial v_{j}}{\partial x_{i}} w_{j} d x .
$$

The continuity property of the trilinear form enables us to define (using Riesz representation Theorem) a bilinear continuous operator $B(u, v) ; V_{2} \times V_{2} \rightarrow V_{2}^{\prime}$ will be defined by

$$
\langle B(u, v), w\rangle=b(u, v, w), \quad \forall w \in V_{2} .
$$

Recall that for $u$ satisfying $\nabla . u=0$ we have

$$
b(u, u, u)=0 \quad \text { and } \quad b(u, v, w)=-b(u, w, v) .
$$

We recall some well known inequalities that we will be using in what follows. Young's inequality

$$
a b \leq \frac{\sigma}{p} a^{p}+\frac{1}{q \sigma^{\frac{q}{p}}} b^{q}, \quad a, b, \sigma>0, \quad p>1, q=\frac{p}{p-1} .
$$

Poincaré's inequality

$$
\lambda_{1}\|u\|^{2} \leq\left\|A^{\frac{1}{2}} u\right\|^{2} \quad \text { for all } u \in V_{0} .
$$

Denoting

$$
\|u\|_{G(t)}^{2}=\left\|e^{t A^{\frac{1}{2}}} u\right\| \quad \text { and } \quad(u, v)_{G(t)}=\left(e^{t A^{\frac{1}{2}}} u, e^{t A^{\frac{1}{2}}} v\right) .
$$

The set $D\left(e^{\alpha A}\right)$ is called the Gevrey class of operator of order $\alpha \geq 0$, see [8]. Our use of Gevrey classes shall be based on the following consideration.

Recall that for a function $h: \Omega \rightarrow \mathbb{R}, N_{h}^{c}=\{x \in \Omega: h(x)=c\}$, and let $\mathcal{H}^{1}$ be the one-dimensional Hausdorff measure operating on subsets of $\mathbb{R}^{2}$.

\section{Level sets of the vorticity function}

Using the operators defined above, we can write (1.1) in the evolution form

$$
\begin{aligned}
\frac{d u}{d t}+\varepsilon A^{p} u+\nu A u+B(u, u) & =f(x), \quad \text { in } \Omega \times(0, \infty), \\
\operatorname{div} u=0 & \text { and } u_{0}(x)=u_{0}, \quad \text { in } \Omega .
\end{aligned}
$$

The existence and uniqueness results for initial value problem (1.1) can be found in [18]. The following theorem collects the main result in this work.

Theorem 3.1. For $p \geq 1$, for $\varepsilon>0$ fixed, $f \in L^{2}\left(0, T ; V_{0}^{\prime}\right)$ and $u_{0} \in V_{0}$ be given, there exists a unique weak solution of (1.1) which satisfies $u \in L^{2}\left(0, T ; V_{p}\right) \cap$ $L^{\infty}\left(0, T ; V_{0}\right), \forall T>0$. 
The modern understanding of turbulence is that it is a collection of weakly correlated vortical motions, which, despite their intermittent and chaotic distribution over a wide range of space and time scales, actually consist of local characteristic 'eddy' patterns that persist as they move around under the influences of their own and other eddies' vorticity fields [12].

In fluid mechanics, the Reynolds number is important in analyzing any type of flow when there is substantial velocity gradient (i.e. shear.). It indicates the relative significance of the viscous effect compared to the inertia effect. The Reynolds number is proportional to inertial force divided by viscous force (see [7])

$$
R e=\frac{U L}{\nu}, \quad U^{2}=L^{-2}\left\langle\|u\|^{2}\right\rangle
$$

where $L$ the characteristic scale of the forcing and $\langle\cdot\rangle$ is the long-timeaverage

$$
\langle g(.)\rangle=\lim _{T \rightarrow \infty} \sup \left(\frac{1}{T} \int_{0}^{T} g(t) d t\right) .
$$

With Reynolds number calculator we can analyze what makes fluid flow regime laminar and what is needed to force the fluid to flow in turbulent regime. Experimental observations show that for 'fully developed' flow, laminar flow occurs when $R e<R_{l} e$ and turbulent flow occurs when $R e>R_{t} e$. In the interval between $R_{l} e$ and $R_{t} e$, laminar and turbulent flows are possible ('transition' flows) [7] and references therein. The nature of the vortex formed in the fluid flow depends strongly on the Reynolds number $([22]$; and references therein). These transition Reynolds numbers are also called critical Reynolds numbers, and were studied by Osborne Reynolds around 1895 [21]. The transition to turbulence and the construction of vortex are delayed by increasing the critical Reynolds number. If we assume that the critical Reynolds number $R_{c} e$ for the onset of vortex shedding is reached for

$$
\|u\|=\nu R_{c} e L
$$

then for each $R e \geq R_{c} e$ the associate velocity $u$ satisfies the inequality

$$
\|u\| \geq \nu R_{c} e L=\mu,
$$

$\mu$ is a positive constant.

Another nondimensional quantity that we use often is the so-called Grashof number, which is proportional to the forcing term $f$. Hence, we define the Grashof numbers in the 2-dimensional case, as in Foias et al. [7] by

$$
G_{r}(f)=\frac{1}{\nu^{2} \lambda_{1}}\|f\|
$$

The effects of variation in Grashof number on vortex have been shown in the work of Olson and Titi [19], they keep the spatial structure of the forcing function fixed and vary the Grashof number by varying the amplitude of the forcing function. Namely, they vary the Grashof number by rescaling the forcing function by a multiplicative factor. This is equivalent to changing the viscosity or the size of the domain. As $G_{r}$ increases, or equivalently as the 
viscosity decreases, the turbulent flow becomes more energetic and one would expect the number of numerically determining modes to increase as well. There are many reasons to suppose that the existence and intensity of vortex in our work should increase as the Grashof number increases [11,19,20]. In [11], zero forcing implies that the attractor has been reduced to zero. Since all solutions decay eventually to zero in the unforced case.

This intuition is supported by existing theoretical critical number $G_{r}^{c}(f)$ for the existence of level curves of representative vorticity fields.

Note the flow for $G_{r}(f) \geq G_{r}^{c}(f)$ has noticeably more large scale structure compared to the flow for $G_{r}(f) \leq G_{r}^{c}(f)$. This is consistent with the energy spectra, where most of the energy is in the lowest modes, that is, in the large spatial scales and eddies when the Grashof number is large [19].

The effect of a body force on the vorticity production and the turbulence generation in a fluid flow is described by the Grashof number.

In addition, we assume without loss of generality that $\|f\|$ is bounded. Than, there exist a maximum Grashof number $G_{r}^{\max }(f)$ and a positive constant $\rho$ such that the body force $f$ satisfies the following inequality

$$
\|f\| \leq \nu^{2} \lambda_{1} G_{r}^{\max }(f)=\rho .
$$

Since $\|f\|$ is strictly positive we get

$$
\frac{\|u\|}{\|f\|} \geq \frac{\mu}{\rho}=\frac{L R_{c} e}{\nu \lambda_{1} G_{r}^{\max }(f)}=\beta,
$$

this gives a relation between $\|u\|$ and $\|f\|$

$$
\|u\| \geq \beta\|f\| .
$$

According to the definition of the Gevrey norm and the relation (3.9) we get

$$
\|u\|_{G} \geq \beta\|f\|_{G} .
$$

The above inequality is valid for the 3D case for some modifications of the Grashof number. The vorticity, $\omega=\nabla \times u$ satisfies the equation

$$
\left(\frac{d}{d t}+u \cdot \nabla+\nu \triangle+\varepsilon(-\triangle)^{p}\right) \omega=\omega \cdot \nabla u+F
$$

where $F=\nabla \times f$.

Theorem 3.2. [14]. Suppose that a nonzero function $h \in V_{1}$ satisfies $\left\|e^{\alpha A} h\right\|_{1} \leq$ $M\|h\|_{1}$, then

$$
\mathcal{H}^{1}\left(N_{h}^{0}\right) \leq C_{1} L^{2}(1+\log M) e^{C_{2} L / \alpha} .
$$

Hereafter, $C_{i}$ for $i \in \mathbb{N}$, stand for universal constants. The above statement will be used in conjunction with the following statement:

Lemma 3.3. [13]. Let $u \in V_{0}$, and let $\omega$ be its vorticity. If

$$
\left\|A^{\frac{1}{2}} e^{\alpha A^{\frac{1}{2}}} u\right\| \leq M\left\|A^{\frac{1}{2}} u\right\|
$$


for some $M>0$, then, for every $c \in \mathbb{R}$

$$
\left\|e^{\alpha A^{\frac{1}{2}}}(\omega-c)\right\|_{1} \leq M\|\omega-c\|_{1} .
$$

For the rest of the paper, let $u(t)$ be an arbitrary solution of the system (1.1).

Theorem 3.4. Let $\|u\| \geq \beta\|f\|$ for any $\alpha \leq \frac{\nu \lambda_{1}^{\frac{1}{2}}}{4}$ and $\beta \leq \frac{4 \sqrt{2}}{\nu}$, then there exists a universal constant $C_{3}$ such that if $\left\|A^{\frac{1}{2}} u\right\| \leq C_{3}$, then

$$
\left\|A^{\frac{1}{2}} e^{\alpha t A^{\frac{1}{2}}} u\right\| \leq 2\left\|A^{\frac{1}{2}} u_{0}\right\|, \quad t \geq 0 .
$$

Proof. For any $\alpha, t \geq 0$, we take the inner product of (3.1) with $u$, to obtain

$$
\begin{aligned}
\frac{1}{2} \frac{d}{d t}\left\|A^{\frac{1}{2}} u\right\|_{G(t)}^{2}= & \alpha\left\|A^{\frac{3}{4}} u\right\|_{G(t)}^{2}+(A \dot{u}, u)_{G(t)} \\
= & \alpha\left\|A^{\frac{3}{4}} u\right\|_{G(t)}^{2}-\varepsilon\left\|A^{\frac{p+1}{2}} u\right\|_{G(t)}^{2} \\
& -\nu\|A u\|_{G(t)}^{2}-b(u, u, A u)_{G(t)}+(f, A u)_{G(t)},
\end{aligned}
$$

then using the Young's inequality (2.5) we have

$$
\begin{aligned}
\frac{1}{2} \frac{d}{d t}\left\|A^{\frac{1}{2}} u\right\|_{G(t)}^{2} \leq & -\varepsilon\left\|A^{\frac{p+1}{2}} u\right\|_{G(t)}^{2}+\frac{\nu}{4}\|A u\|_{G(t)}^{2}+\frac{\alpha^{2}}{\nu}\left\|A^{\frac{1}{2}} u\right\|_{G(t)}^{2}-\nu\|A u\|_{G(t)}^{2} \\
& +\left(\frac{\nu}{2}\|A u\|_{G(t)}^{2}+\frac{1}{2 \nu}\|f\|_{G(t)}^{2}\right)+b(u, u, A u)_{G(t)} .
\end{aligned}
$$

Applying the Poincaré's inequality we get

$$
\begin{aligned}
\frac{1}{2} \frac{d}{d t}\left\|A^{\frac{1}{2}} u\right\|_{G(t)}^{2} \leq & -\varepsilon\left\|A^{\frac{p+1}{2}} u\right\|_{G(t)}^{2}+\frac{\nu}{4}\|A u\|_{G(t)}^{2}+\frac{\alpha^{2}}{\nu}\left\|A^{\frac{1}{2}} u\right\|_{G(t)}^{2}-\nu\|A u\|_{G(t)}^{2} \\
& +\left(\frac{\nu}{2}\|A u\|_{G(t)}^{2}+\frac{\beta^{2}}{2 \lambda_{1} \nu}\left\|A^{\frac{1}{2}} u\right\|_{G(t)}^{2}\right)+b(u, u, A u)_{G(t)} \\
\leq & -\varepsilon\left\|A^{\frac{p+1}{2}} u\right\|_{G(t)}^{2}+\frac{-\lambda_{1} \nu}{4}\left\|A^{\frac{1}{2}} u\right\|_{G(t)}^{2}+\frac{\alpha^{2}}{\nu}\left\|A^{\frac{1}{2}} u\right\|_{G(t)}^{2} \\
& +\frac{\beta^{2}}{2 \lambda_{1} \nu}\left\|A^{\frac{1}{2}} u\right\|_{G(t)}^{2}+b(u, u, A u)_{G(t)} .
\end{aligned}
$$

We get for $\beta^{2}=\frac{1}{2 \lambda_{1} \alpha^{2}}$

$$
\begin{aligned}
\frac{1}{2} \frac{d}{d t}\left\|A^{\frac{1}{2}} u\right\|_{G(t)}^{2} \leq & -\varepsilon\left\|A^{\frac{p+1}{2}} u\right\|_{G(t)}^{2}-\frac{\lambda_{1} \nu}{4}\left\|A^{\frac{1}{2}} u\right\|_{G(t)}^{2} \\
& +\frac{2 \alpha^{2}}{\nu}\left\|A^{\frac{1}{2}} u\right\|_{G(t)}^{2}+b(u, u, A u)_{G(t)} .
\end{aligned}
$$

We use the following inequality from [8] and [13, Section 4]

$$
b(u, u, A u)_{G(t)} \leq C_{4}\left\|A^{\frac{1}{2}} u\right\|_{G(t)}^{2}\|A u\|_{G(t)}\left(1+\log \frac{\|A u\|_{G(t)}^{2}}{\lambda_{1}\left\|A^{\frac{1}{2}} u\right\|_{G(t)}^{2}}\right)^{\frac{1}{2}}
$$


to obtain

$$
\begin{aligned}
\frac{1}{2} \frac{d}{d t}\left\|A^{\frac{1}{2}} u\right\|_{G(t)}^{2} \leq & -\varepsilon\left\|A^{\frac{p+1}{2}} u\right\|_{G(t)}^{2}-\frac{\lambda_{1} \nu}{4}\left\|A^{\frac{1}{2}} u\right\|_{G(t)}^{2}+\frac{2 \alpha^{2}}{\nu}\left\|A^{\frac{1}{2}} u\right\|_{G(t)}^{2} \\
& +C_{4}\left\|A^{\frac{1}{2}} u\right\|_{G(t)}^{2}\|A u\|_{G(t)}\left(1+\log \frac{\|A u\|_{G(t)}^{2}}{\lambda_{1}\left\|A^{\frac{1}{2}} u\right\|_{G(t)}^{2}}\right)^{\frac{1}{2}}
\end{aligned}
$$

To establish (3.14) we use the estimate [13]

$$
a \mu\left(1+\log \frac{\mu^{2}}{b^{2}}\right)^{\frac{1}{2}} \leq d \mu^{2}+\frac{a^{2}}{d^{2}} \log \frac{2 a}{b d} \quad a, d>0, \mu \geq b>0 .
$$

By applying the Poincaré's inequality (2.6), we have that for $\mu=\|A u\|_{G(t)}$ and $d=\frac{\nu}{8}$

$$
\begin{aligned}
\frac{1}{2} \frac{d}{d t}\left\|A^{\frac{1}{2}} u\right\|_{G(t)}^{2}+\varepsilon\left\|A^{\frac{p+1}{2}} u\right\|_{G(t)}^{2} \leq & -\frac{\lambda_{1} \nu}{8}\left\|A^{\frac{1}{2}} u\right\|_{G(t)}^{2}+\frac{2 \alpha^{2}}{\nu}\left\|A^{\frac{1}{2}} u\right\|_{G(t)}^{2} \\
& +C_{5}\left\|A^{\frac{1}{2}} u\right\|_{G(t)}^{4} \log \frac{C_{6}\left\|A^{\frac{1}{2}} u\right\|_{G(t)}}{\lambda_{1}^{\frac{1}{2}}}
\end{aligned}
$$

Letting $\alpha \leq \frac{\nu \lambda_{1}^{\frac{1}{2}}}{4}$ we have for $\beta \leq \frac{4 \sqrt{2}}{\nu}$ that

$$
\frac{1}{2} \frac{d}{d t}\left\|A^{\frac{1}{2}} u\right\|_{G(t)}^{2} \leq C_{5}\left\|A^{\frac{1}{2}} u\right\|_{G(t)}^{4} \log \frac{C_{6}\left\|A^{\frac{1}{2}} u\right\|_{G(t)}}{\lambda_{1}^{\frac{1}{2}}} .
$$

If $\left\|A^{\frac{1}{2}} u\right\|<\frac{\lambda_{1}^{\frac{1}{2}}}{C_{6}}=C_{3}$, the term with a logarithm in (3.18) is negative, and thus (3.18) implies that $\left\|A^{\frac{1}{2}} u\right\|_{G(t)}$ is a decreasing function of $t$.

Let $\Omega$ be a periodic box, for simplicity assume $\Omega=(0, L)^{2}, A$ has eigenvalues $0<\lambda_{1}<\lambda_{2}<\ldots$ with corresponding eigenspaces $E_{1}, E_{2}, \ldots$ Let $P_{m}$ be the projection on the eigenspaces $E_{1} \oplus E_{2} \oplus \cdots \oplus E_{m}$ and let $Q_{m}=I-P_{m}$ we have $\|u\|^{2}=\left\|P_{m} u\right\|^{2}+\left\|Q_{m} u\right\|^{2}$ and we also have from (2.2) that

$$
\left\|A^{p} u\right\| \leq \lambda_{m}^{p}\|u\| \quad \text { for every } p \geq 0 \text { and } u \in D\left(A^{p}\right) \text {. }
$$

For any $t \geq 0$, let $\omega(t)$ be the vorticity of $u(t)$. We shall, for any fixed $t>0$, estimate the quantity

$$
\ell(\omega(t))=\sup _{c \in \mathbb{R}} \mathcal{H}_{p}^{1}\left(N_{\omega}^{c}\right) .
$$

Lemma 3.5. Let $\|u\| \geq \beta\|f\|$ for any $\beta \geq 0$, then

$$
\|u(t)\| \geq\|u(0)\| \exp (\eta t) \text { for every } t \geq 0
$$

with $\eta=-\left(\varepsilon \lambda_{m}^{\frac{p}{2}}+\frac{1+\beta^{2}}{2 \beta^{2}}\right)$. 
Proof. Taking the scalar product of both sides of (1.1) by $u(t)$ and using (2.4), we have that

$$
\frac{1}{2} \frac{d}{d t}\|u\|^{2}+\nu\left\|A^{\frac{1}{2}} u\right\|^{2}+\varepsilon\left\|A^{\frac{p}{2}} u\right\|^{2}=(f, u) \quad \text { for } t \geq 0 .
$$

Using (3.19) and the following inequality

$$
(f, u) \geq-\frac{1}{2}\|f\|^{2}-\frac{1}{2}\|u\|^{2} .
$$

Because the increasing sequence $0 \leq \lambda_{1} \leq \lambda \leq \lambda_{m}$ we have

$$
\frac{1}{2} \frac{d}{d t}\|u\|^{2} \geq-\nu\left\|A^{\frac{1}{2}} u\right\|^{2}-\left(\varepsilon \lambda_{m}^{\frac{p}{2}}+\frac{1+\beta^{2}}{2 \beta^{2}}\right)\|u\|^{2}
$$

note that since $\left\|A^{\frac{1}{2}} u\right\| \leq C_{3}$ we have that

$$
\frac{1}{2} \frac{d}{d t}\|u\|^{2} \geq-\nu C_{3}-\left(\varepsilon \lambda_{m}^{\frac{p}{2}}+\frac{1+\beta^{2}}{2 \beta^{2}}\right)\|u\|
$$

if we set $\eta=-\left(\varepsilon \lambda_{m}^{\frac{p}{2}}+\left(1+\beta^{2}\right) / 2 \beta^{2}\right)$, then we have from (3.25) that

$$
\frac{1}{2} \frac{d}{d t}\|u\|^{2} \geq-\nu C_{3}+\eta\|u\| \text {. }
$$

Integrating the above inequality from 0 to $t$, we get

$$
\|u\|^{2} \geq \frac{-\nu C_{3}}{\eta}(1-\exp (\eta t))+\|u(0)\|^{2} \exp (\eta t)
$$

or, since $\frac{-\nu C_{3}}{\eta}(1-\exp (\eta t)) \geq 0$. Thus, we have the inequality (3.21).

Proposition 3.6. Let $\|u\| \geq \beta\|f\|$ and $u_{0} \neq 0$, and suppose that $\left\|u_{0}\right\| \leq C_{3} \nu \lambda_{1}^{\frac{1}{2}}$, then we have that

$$
\ell(\omega(t)) \leq C_{1} L\left(1+\frac{1}{2} \log \frac{\lambda_{m}}{\lambda_{1}}+\left(\varepsilon \lambda_{m}^{\frac{p}{2}}+\frac{1+\beta^{2}}{2 \beta^{2}}\right) t\right) e^{\frac{C_{2} L}{\alpha t}} \text { for } t \geq 0,
$$

for any $\alpha \leq \frac{\nu \lambda_{1}^{\frac{1}{2}}}{4}$ and $\beta \leq \frac{4 \sqrt{2}}{\nu}$.

Proof. By Theorem 3.4 and Lemma 3.5, we get for $t \geq 0$ the following

$$
\begin{aligned}
\left\|A^{\frac{1}{2}} e^{\alpha t A^{\frac{1}{2}}} u\right\| & \leq 2\left\|A^{\frac{1}{2}} u_{0}\right\| \\
& \leq 2 \lambda^{\frac{1}{2}}\left\|u_{0}\right\|
\end{aligned}
$$

and use the inequality (3.21) to get

$$
\begin{aligned}
\left\|A^{\frac{1}{2}} e^{\alpha t A^{\frac{1}{2}}} u\right\| & \leq 2 \lambda^{\frac{1}{2}}\|u(t)\| \exp \left(\varepsilon \lambda_{m}^{\frac{p}{2}}+\frac{1+\beta^{2}}{2 \beta^{2}}\right) t \\
& \leq 2\left(\frac{\lambda}{\lambda_{1}}\right)^{\frac{1}{2}}\left\|A^{\frac{1}{2}} u(t)\right\| \exp \left(\varepsilon \lambda_{m}^{\frac{p}{2}}+\frac{1+\beta^{2}}{2 \beta^{2}}\right) t .
\end{aligned}
$$

The rest follows by combining (3.29) with Lemma 3.3 and Theorem 3.2.

The foundational result for our one-dimensional Hausdorff measure estimates of the level sets of the vorticity of solutions is 
Theorem 3.7. Let $\|u\| \geq \beta\|f\|, u_{0} \neq 0$, and suppose that $\left\|u_{0}\right\| \leq C_{3} \nu \lambda_{1}^{\frac{1}{2}}$, then

$$
\ell(\omega(t)) \leq C_{7} \lambda_{m}^{\frac{p}{2}} \text { for } t \geq t_{0},
$$

with $t_{0}=\frac{2 C_{2} L}{\nu \lambda_{1}^{\frac{1}{2}}}$ for any $\alpha \leq \frac{\nu \lambda_{1}^{\frac{1}{2}}}{4}$ and $\beta \leq \frac{4 \sqrt{2}}{\nu}$.

Proof. For $t \geq 2 C_{2} L / \nu \lambda_{1}^{\frac{1}{2}}$ the inequality (3.27) implies

$$
\ell(\omega(t)) \leq C_{1} e\left(2+\frac{1}{2} \log \frac{\lambda_{m}}{\lambda_{1}}+\left(\varepsilon \lambda_{m}^{\frac{p}{2}}+\frac{1+\beta^{2}}{2 \beta^{2}}\right) \frac{C_{2} L}{\alpha}\right) \quad \text { for } t \geq 0 .
$$

Since $\lambda_{m} \geq \lambda_{1}$, (3.30) follows directly from the above inequality.

The estimate of $\mathcal{H}_{p}^{1}$ grows in $m$ at a rate lower than $\frac{p}{2}$.

Proposition 3.8. Let $\|u\| \geq \beta\|f\|$ and $u_{0} \neq 0$, and suppose that $\left\|u_{0}\right\| \leq C_{3} \nu \lambda_{1}^{\frac{1}{2}}$, then

$$
\sup _{t \rightarrow \infty} \ell(\omega(t)) \leq C_{8} m^{\frac{p}{2}} \quad \text { for } t \geq t_{0}
$$

for any $\alpha \leq \frac{\nu \lambda_{1}^{\frac{1}{2}}}{4}$ and $\beta \leq \frac{4 \sqrt{2}}{\nu}$.

Proof. Note that in the $2 \mathrm{D}$ case we have $\lambda_{j} \geq C_{9} \lambda_{1} j$ for some positive universal constant (see, for example [23, Lemma VI 2.1]). Therefore, since $\lambda_{m} \backsim \lambda_{1} m$ the growth in $m$ of the Hausdorff measure (3.32) is less than $m^{\frac{p}{2}}$.

If we impose the condition $\lambda_{m} \leq\left(\frac{1}{l_{\epsilon}}\right)^{2}$ or $\frac{\lambda_{m}}{\lambda_{1}} \leq\left(\frac{l_{0}}{l_{\epsilon}}\right)^{2}$ where $l_{0}=\lambda_{1}^{\frac{-1}{2}}$ represents characteristic macroscopic length, and $l_{\epsilon}$ is the Kolmogorov length scale, i.e. $l_{\epsilon}=\nu^{\frac{3}{4}} \epsilon^{\frac{-1}{4}}$ where $\epsilon$ is Kolmogorov's mean rate of dissipation of energy in turbulent flow (see e.g. $[1,7,23]$, and the references contained therein) is defined as

$$
\epsilon=\nu \lambda_{1} \lim \sup _{t \rightarrow \infty} \frac{1}{t} \int_{0}^{t}\left\|A^{\frac{1}{2}} u\right\|^{2} d s .
$$

Substituting this in (3.30) gives

$$
\sup _{t \rightarrow \infty} \ell(\omega(t)) \leq C_{10}\left(\frac{l_{0}}{l_{\epsilon}}\right)^{p} .
$$

Since the (dimensionless) Grashoff number $G_{r}$ (see e.g. $[1,7,23]$ ) is an upper bound for $\left(\frac{l_{0}}{l_{\epsilon}}\right)^{2}$. Hence, we obtain for the Hausdorff measure of the equation (1.1) the following estimate in terms of the Grashoff number $G_{r}$.

Proposition 3.9. Let $\|u\| \geq \beta\|f\|$ and $u_{0} \neq 0$, and suppose that $\left\|u_{0}\right\| \leq C_{3} \nu \lambda_{1}^{\frac{1}{2}}$, then for any $\alpha \leq \frac{\nu \lambda_{1}^{\frac{1}{2}}}{4}$ and $\beta \leq \frac{\nu \lambda_{1}}{2 \sqrt{2}}$ we have

$$
\sup _{t \rightarrow \infty} \ell(\omega(t)) \leq C_{11} G_{r}^{\frac{p}{2}} \quad \text { for } t \geq t_{0} .
$$


This result holds independently of $m$. The estimate grows in $p$ at a rate lower than $\frac{p}{2}$. If we impose $p=1$, the estimates become $\sup _{t \rightarrow \infty} \ell(\omega(t)) \leq$ $C_{11} G_{r}^{\frac{1}{2}}$. This result recover the usual 2D Navier-Stokes equations estimates. Here again our results indicate that under certain conditions the upper bounds for $\mathcal{H}_{p}^{1}\left(N_{\omega}^{0}\right)$ converge to the associated upper bounds of the Hausdorff measure $\mathcal{H}_{1}^{1}\left(N_{\omega}^{0}\right)$ estimates for the usual 2D Navier-Stokes equations with potential force.

Proving global regularity for the Navier-Stokes equations is one of the most challenging outstanding problems in nonlinear analysis. The main difficulty in establishing this result lies in controlling certain norms of vorticity. More specifically, the vorticity stretching term in the 3D vorticity equation forms the main obstacle to achieving this control, Foias [6] and estimates on the number of degrees of freedom for the Navier-Stokes equations and its closure models are a measure of the complexity of the system, Avrin [1]. This paper proposed another way to estimate this complexity through bounding the size of the nodal set for the vorticity and expressing this estimate in terms of $G_{r}$. This result answering a interesting question raised by Avrin in 2D [1]. This paper gives an overview of the foundational issues in the $3 \mathrm{D}$ case.

Another interesting way to study decaying turbulence in the hyperviscous flow is to provide a numerical investigation of our theoretical results on the dependence of turbulence structure and vortex dynamics, as was done in [19] for the number of numerically determining modes in the 2D Navier-Stokes equations.

\section{References}

[1] Avrin, J.: The asymptotic finite-dimensional character of a spectrally-hyperviscous model of 3D turbulent flow. J. Dyn. Diff. Eqn. 20, 1-40 (2008)

[2] Borue, V., Orszag, S.: Numerical study of three-dimensional Kolmogorov flow at high Reynolds numbers. J. Fluid Mech. 306, 293-323 (1999)

[3] Borue, V., Orszag, S.: Local energy flux and subgrid-scale statistics in threedimensional turbulence. J. Fluid Mech. 306, 1-31 (1998)

[4] Constantin, P.: Navier Stokes equations and area of interfaces. Commun. Math. Phys. 129, 241-266 (1990)

[5] Donnelly, H., Fefferman, C.: Nodal sets of eigenfunctions: Riemannian manifolds with boundary, Moser Volume, analysis, pp 251-262. Academic Press, Boston (1990)

[6] Foias, C., Holm, D.D., Titi, E.S.: The three dimensional viscous Camassa-Holm equations, and their relation to the Navier-Stokes equations and turbulence theory. J. Dyn. Differ. Equ. 14(1), 1-35 (2002)

[7] Foias, C., Manley, O., Rosa, R., Temam, R.: Navier-Stokes equations and turbulence. Cambridge University Press, Cambridge (2001) 
[8] Foias, C., Temam, R.: Gevrey class regularity for the solutions of the Navier Stokes equations. J. Funct. Anal. 87, 359-369 (1989)

[9] Han, Q., Lin, F.H.: On the geometric measure of nodal sets of solutions. J. Partial Differ. Equ. 7, 111-131 (1994)

[10] Hardt, R., Simon, L.: Nodal sets of solutions of elliptic equation. J. Differ. Geom. 30, 505-522 (1989)

[11] Henshaw, W.D., Kreiss, H.O., Yström, J.: Numerical experiments on the interaction between the large and small-scale motions of the Navier-Stokes equations. Multisc. Model. Simul. 1, 119-149 (2003)

[12] Hunt, J.C.R., Vassilicos, J.C.: Turbulence structure and vortex dynamics. Cambridge University Press, Cambridge (2000)

[13] Kukavica, I.: Level sets of the vorticity and the stream function for the 2D periodic Navier-Stokes equations with potential forces. J. Differ. Equ. 126, 374388 (1995)

[14] Kukavica, I.: Hausdorff length of level sets for solutions of the Ginzburg Landau equation. Nonlinearity 8, 113-129 (1995)

[15] Kukavica, I.: Nodal volumes for eigenfunctions of analytic regular elliptic problems. J. Anal. Math. 67, 269-280 (1995)

[16] Leray, J.: Sur le mouvement d'un liquide visqueux emplissant l'espace. Acta Math. 63, 193-248 (1934)

[17] Lin, F.H.: Nodal sets of solutions of elliptic and parabolic equations. Commun. Pure Appl. Math. 44, 287-308 (1991)

[18] Lions, J.L.: Quelques Méthodes de Résolution des Problèmes aux Limites Non Linéaires. Dunod Gauthier-Villars, Paris (1969)

[19] Olson, E., Titi, E.S.: Determining modes and Grashof number in 2D turbulence - a numerical case study. Theor. Comput. Fluid Dyn. 22(5), 327-339 (2008)

[20] Perot, J.B.: Turbulence modeling using body force potentials. Phys. Fluids. 11(9) (1999)

[21] Reynolds, O.: On the experimental investigation of the circumstances which determine whether the motion of water shall be direct or sinuous. Phil. Trans. R. Soc. Lond. Ser. A 74, 935-982 (1883)

[22] Sassa, K., Makita, H.: Reynolds number dependence of elementary vortices in turbulence. In: Engineering Turbulence Modelling and Experiments, vol. 6. pp. 431-440. Elsevier, Oxford (2005)

[23] Temam, R.: Infinite-Dimensional Dynamical Systems in Mechanics and Physics, Applied Mathematical Sciences Series, 68. 2nd edn. Springer, New york (1997)

[24] Younsi, A.: Effect of hyperviscosity on the Navier-Stokes turbulence. Electron. J. Diff. Equ. 110, 1-19 (2010) 


\author{
Abdelhafid Younsi \\ Department of Mathematics and Computer Science \\ University of Djelfa \\ Djelfa \\ Algeria \\ e-mail: younsihafid@gmail.com
}

Received: 27 August 2011.

Accepted: 8 May 2012. 